See discussions, stats, and author profiles for this publication at: https://www.researchgate.net/publication/243491501

\title{
Rotational Raman scattering in the instructional laboratory
}

Article in American Journal of Physics · July 1994

DOI: 10.1119/1.17484

\section{CITATIONS}

12

3 authors, including:

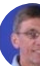

Alvin D. Compaan
University of Toled

286 PUBLICATIONS 3,344 CITATIONS

SEE PROFILE

Some of the authors of this publication are also working on these related projects:

Project Development of High Efficiency CdTe solar cells View project

\section{READS}

25 
Now, the initial state of our detector will be subject to the Heisenberg relation

$$
\Delta P \Delta X \geqslant \frac{\hbar}{2},
$$

so that, in order for $\Delta m$ to be "noticeable" by the detector, the impulse $P$ will have to be bigger than $2 \Delta P$, since the initial momentum of $M$ lies, with probability $\frac{2}{3}$, say, in the range $-\Delta P$ to $+\Delta P$. Then, from Eqs. (2) and (3), we have

$$
\Delta X>\frac{2 R^{2} c^{2}}{G M}=4 R\left(\frac{R}{R_{S}}\right),
$$

where $R_{S}$ is the Schwartzschild radius of our detector $M$, given by $2 G M / c^{2}$. Now, for objects ranging in size from protons to planets, $R_{S}$ lies well within the object itself. Indeed, in the case of an electron (or a proton) it is many orders of magnitude smaller than the Planck length. It would seem safe to conclude that for any detector, $R \gg R_{S}$, so that $\Delta X$, the indeterminacy in the initial position of the detector, will be much greater than the separation of the detector and the pion, and the momentum transfer will not be observable. (Although we realize that our Newtonian analysis will clearly not apply to the interior of a black hole, it is interesting to observe that for $R \ll R_{S}$, the momentum transfer would be, in principle, observable. This we mention without comment.)

Although the free-particle detector discussed here is not the only one imaginable, it is doubtless the most sensitive. It is therefore clear that gravitational radiation from any vacuum fluctuation is, accordingly, unobservable. It is interesting that the same cannot be said for the electromagnetic analogue in the case of, say, an electron-positron pair fluctuation $^{3}$ as there is no electromagnetic counterpart of the Schwartzschild radius.

${ }^{1}$ H. Yukawa, "Interaction of elementary particles. Part I," Proc. Phys. Math. Soc. Jpn. 17, 48-57 (1935).

${ }^{2}$ H. G. B. Casimir, "On the attraction between two perfectly conducting plates," Proc. K. Ned. Akad. Wet. (Amsterdam) 51, 793-796 (1948).

${ }^{3}$ S. W. Hawking, "Particle creation by black holes," Commun. Math. Phys. 43, 199-219 (1975).

${ }^{4}$ C. J. Isham, "An introduction to quantum gravity," Quantum Gravity: An Oxford Symposium, edited by C. J. Isham, R. Penrose, and D. W. Sciama (Clarendon, Oxford, 1975), p. 1-77.

${ }^{5}$ C. J. Isham, "Quantum gravity," in The New Physics, edited by P. C. W. Davies (Cambridge University, Cambridge, 1989), pp. 70-93.

${ }^{6}$ S. W. Hawking, "Quantum cosmology," in 300 Years of Gravitation, edited by S. W. Hawking and W. Israel (Cambridge University, Cambridge, 1987), pp. 631-651.

\title{
Rotational Raman scattering in the instructional laboratory
}

\author{
A. Compaan and A. Wagoner \\ Department of Physics and Astronomy, The University of Toledo, Toledo, Ohio 43606
}

\author{
A. Aydinli \\ Department of Physics, Bilkent University, Ankara, Turkey
}

(Received 13 August 1993; accepted 4 November 1993)

\begin{abstract}
We describe the use of a single spectrometer and a low power argon laser for the study of the pure rotational Raman spectra of $\mathrm{H}_{2}, \mathrm{O}_{2}, \mathrm{~N}_{2}$, and $\mathrm{CO}_{2}$. Analyses of the spectra allow the student to obtain internuclear separations in the ground state, the rotational temperature of the gas, and the influence of nuclear spin on the allowed rotational states. The four molecules chosen for study have nuclei with spins of $I=0\left(\mathrm{O}_{2}\right), 1 / 2\left(\mathrm{H}_{2}\right)$, and $1\left(\mathrm{~N}_{2}\right)$ and thus the discussion includes the effects of both Bose and Fermi exchange properties on the symmetry of the overall wave function. To fully illustrate the range of information available from the data, we provide spectra obtained with a high quality double spectrometer which allow even the lowest rotational states to be observed and show the stretching of the $\mathrm{H}_{2}$ molecule due to centripetal forces.
\end{abstract}

\section{INTRODUCTION}

Although Raman scattering has become a very important spectroscopic tool in research and industrial labs, ${ }^{1}$ it is rare for either undergraduate or graduate students to receive hands-on experience with this technique in an instructional lab. This is largely due to the high cost of double or triple spectrometers, which are the instruments of choice for general purpose Raman scattering, and to the cost of high power $\mathrm{cw}$ laser sources. These instruments are often needed because the very weak Raman scattering requires substantial laser power and furthermore the Raman lines are often ob- scured by strong elastic scattering. The elastic scattering may be $10^{6}-10^{14}$ times stronger than the Raman scattering.

However, gases do not usually create such stray light difficulties and if a scattering cell is carefully constructed to minimize stray light scattering from windows and from the cell interior, Raman scattering can be observed to within a few wave numbers (angstroms) of the laser line. The pioneering and extensive work of Herzberg, ${ }^{2}$ and more recently by Weber, ${ }^{3}$ showed that Raman scattering can yield abundant information on molecular systems including internuclear separations, ground state symmetries, nuclear spins, and force constants. We describe here a system that can be imple- 


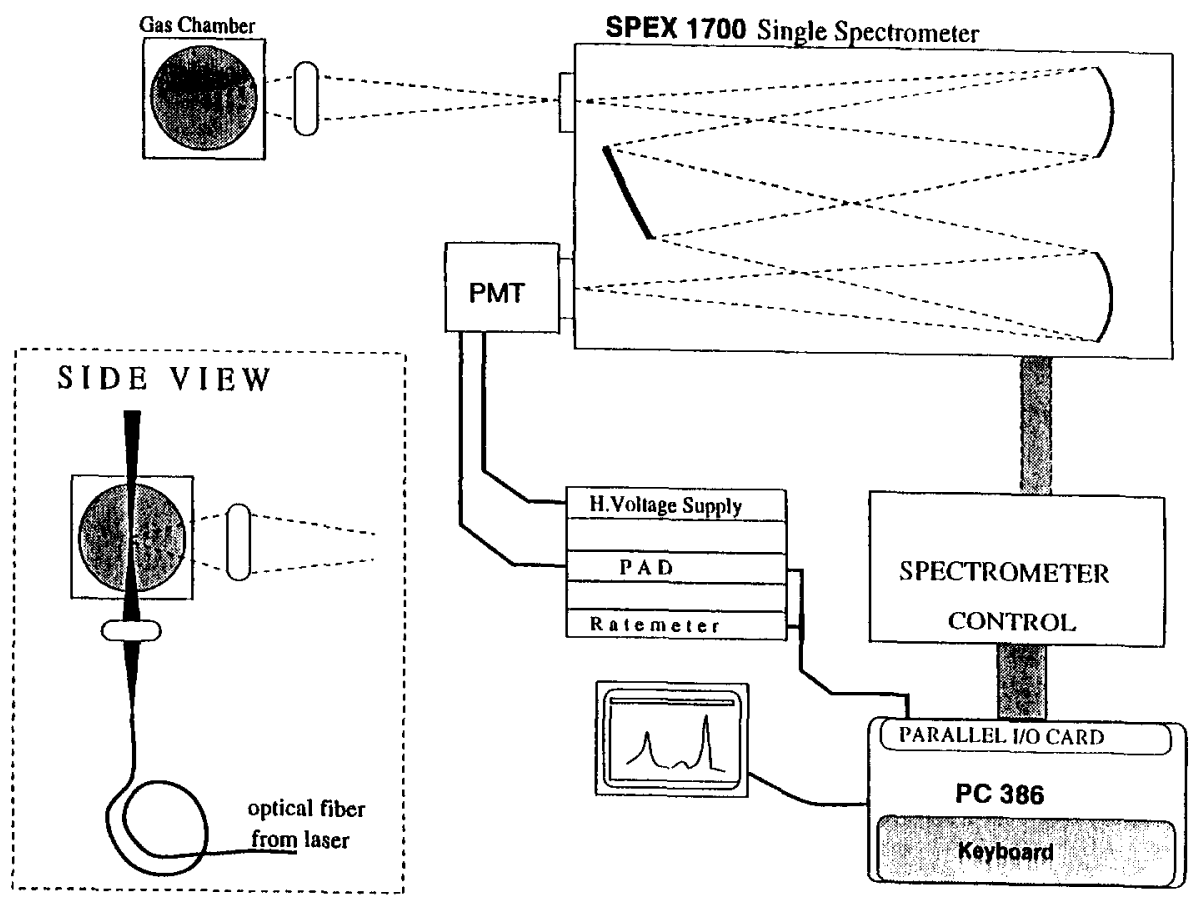

Fig. 1. Experimental arrangement for pure rotational Raman measurements using a single grating spectrometer.

mented in an instructional lab and can make available to students hands-on opportunities to study many of the basic elements of molecular structure.

Pedagogically, the pure rotational Raman spectra of simple gases are ideal in allowing the student to start from the rotational energy of a simple quantized rigid rotator and then proceed to the more complex issues of intensities and of the influence of the nuclear spin on the molecular wave function. Along the way the student can obtain: (1) the molecular internuclear distance in the ground state; (2) the rotational temperature from the line intensities; (3) for the case of $\mathrm{H}_{2}$, the stretching of the molecule due to rotation; and (4) the nuclear spins and their influence on the allowed rotational states via the symmetry requirement on the overall wave function under exchange of the nuclei. It may surprise the student that the effects of nuclear spin on molecular states may be so dramatic that half of the lines of the $\mathrm{O}_{2}$ and $\mathrm{CO}_{2}$ spectra are missing. The student will also learn to appreciate the difference between the exchange properties of bosons ( $\mathrm{O}$ or $\mathrm{N}$ nuclei) and fermions ( $\mathrm{H}$ nuclei).

\section{INSTRUMENTATION}

Two instruments are central to this experiment-a visible wavelength laser with power of $50-100 \mathrm{~mW}$ on a single transition, and a high quality, single grating spectrometer. For the spectrometer we used a $1 \mathrm{~m}$ SPEX 1701 with 1800 $\ell / \mathrm{mm}$ diffraction grating ${ }^{4}$ and stepper motor scan control. (The single spectrometer prevented us from clearly identifying the lowest rotational transitions and therefore, for pedagogical reasons, we also present data obtained with a high quality double spectrometer.) The laser beam was brought into the instructional lab via a multimode optical fiber (200 $\mu \mathrm{m}$ core) from a $5 \mathrm{~W}$, water-cooled argon laser (Lexel $5000)^{5}$ about $70 \mathrm{~m}$ away. Alternatively an air-cooled argon laser would be perfectly adequate for this experiment. The focusing from the multimode optical fiber into the center of the scattering cell was poor, allowing us to obtain a spot size of no less than $400 \mu \mathrm{m}$. (A $50 \mu \mathrm{m}$ or smaller spot size is desirable, as discussed below.) However, the single spectrometer gives significantly higher throughput than double or triple spectrometers and thus the Raman signal was easily detected even with poor laser beam focusing.

The Raman system is sketched in Fig. 1. A camera lens with low $f$ number allows one to collect the Raman light with a large collection angle and image the focused laser beam line into the entrance slit of the spectrometer, matching the spectrometer collection angle. In our case we used a lens with $F=55 \mathrm{~mm}, f / 1.4$ and adjusted the placement of the scattering cell so that the collection angle into the entrance slit matched the spectrometer aperture $(f / 8)$. Note that with a magnification of about 6 , the laser beam waist of about 400 $\mu \mathrm{m}$ was magnified to about $2.4 \mathrm{~mm}$ on the entrance slit. However, achieving sufficient resolution required the use of slit widths of about $200 \mu \mathrm{m}$ for $\mathrm{O}_{2}$ and $\mathrm{N}_{2}$ and about $75 \mu \mathrm{m}$ for $\mathrm{CO}_{2}$. Thus, we estimate that only $10 \%$ or less of the Raman light collected by the lens actually entered the spectrometer. (A beam waist of 20-30 $\mu \mathrm{m}$, easily achieved with a $10 \mathrm{~cm}$ lens focusing a laser beam directly, would yield a corresponding increase in signal.)

The scattered light was detected with a high gain photomultiplier (Thorn-EMI 9635B) ${ }^{6}$ with S-5 photocathode response suitable for photon counting. This photocathode has little red response (work function $\sim 2 \mathrm{eV}$ ) and therefore the photomultiplier can be operated at room temperature with dark counts of only $5-10 / \mathrm{s}$. The rest of the detection electronics were quite standard. We employed a preampamplifier-discriminator (PAD) (Mechtronics 509) ${ }^{7}$ in a NIM module together with a ratemeter (Mechtronics 777) for con- 
venient analog readout independent of the computer. The signal from the PAD was also fed into a standard multiport input card (PCLabCard) ${ }^{8}$ in an 80386 computer.

The scattering cell was not elaborate but was constructed with the idea of minimizing stray light. The cell was machined from a 1.5 in. cube of aluminum with the interior milled out from two directions with a 1.00-in.-diam tool. Seats were machined for the 1.125-in.-diam windows which were sealed with household silicone seal. Before sealing on the windows, the interior of the cell was painted black and onto the interior surface opposite the scattering window we glued a piece of black felt cloth for further suppression of the elastic scattering from the laser entrance and exit windows. We found that sufficient gas purity was obtained without evacuation by simply flushing the cell for a minute or so with the gas of interest before closing the entrance and exit valves on the cell. The cell was designed for filling with gas only at atmospheric pressure. (Higher pressures will increase the signal, of course, but we believe that this would unnecessarily compromise student safety.)

The alignment of the beam vertically through the gas cell requires some care since the image of the beam through the collecting lens onto the vertical entrance slit of the spectrometer is not visible to the eye. Therefore we used a small piece of metal to intercept the laser at the position of the center of the scattering cell (with the cell removed). This provided a bright image to adjust the focusing lens. Usually when the cell was replaced a reasonable signal could be seen, however, it was generally necessary to refocus the collection lens by a millimeter or so to account for the additional optical path of the front window of the cell. This optimization was done with the spectrometer set to one of the stronger Raman lines and using the ratemeter for analog feedback to the operator.

\section{RESULTS WITH THE SINGLE SPECTROMETER}

Figure 2 shows the results obtained on the single spectrometer system described above. For comparison we show two cases: diatomic oxygen and carbon dioxide. The rigid rotator spectrum for $\mathrm{CO}_{2}$ is very similar to that of $\mathrm{O}_{2}$. Since the carbon atom, in this linear molecule, sits at the center of mass, it does not contribute to the rotational inertia. However, the larger internuclear separation is dramatically illustrated in the much smaller line separations. One aspect of these spectra is not immediately apparent; that is the absence of every second line in the spectrum. In fact for $\mathrm{O}_{2}$ only odd values of the rotational angular momentum quantum number, $j$, appear while for $\mathrm{CO}_{2}$ only even values of $j$ appear. This is intimately related to the required symmetry of molecular wave functions under the operation of exchanging the (spin 0) oxygen nuclei (bosons). See the discussion under Analysis.

In order to calibrate the frequency shifts and yet avoid saturating the photomultiplier, we scanned continuously through the laser line position by inserting a neutral density filter which attenuated the scattered beam by $10^{-4}$ for a few wave numbers $\left(\mathrm{cm}^{-1}\right)$ around the laser peak. It should be noted that in the spectra of Fig. 2(b), the sloping baseline extending on either side of the laser peak arises from incomplete rejection of the elastic peak. We have been successful in removing this background by subtracting an appropriately scaled elastic scattering signal obtained from a clean aluminum plate, which has no observable Raman lines. This has
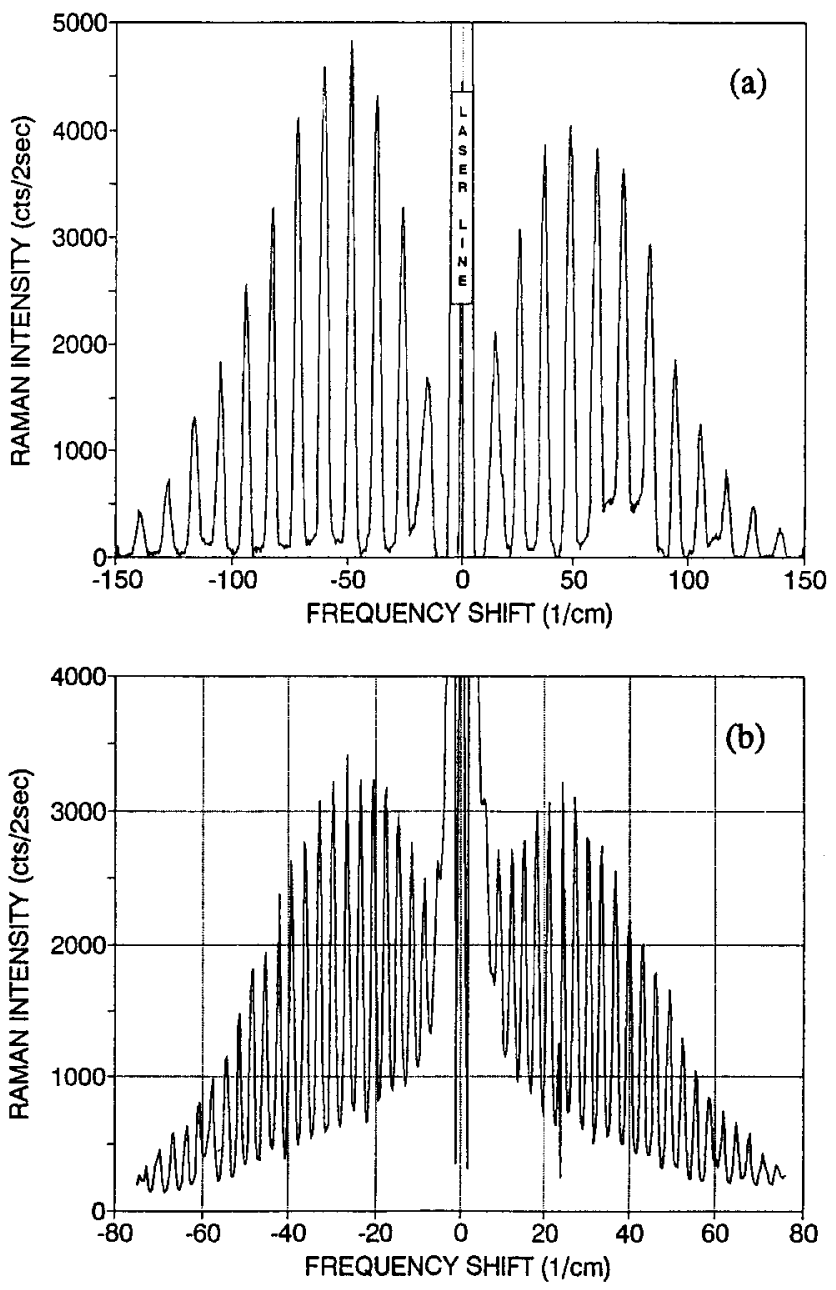

Fig. 2. Rotational Raman spectra obtained with $50 \mathrm{~mW}$ at $514.5 \mathrm{~nm}$ from gas at $1 \mathrm{~atm}$ and a single grating spectrometer. (a) $\mathrm{O}_{2}$ using $150 \mu \mathrm{m}$ slits, (b) $\mathrm{CO}_{2}$ using $75 \mu \mathrm{m}$ slits. For (a) the tail of the elastic peak has been subtracted. Some $\mathrm{N}_{2}$ contamination is evident near $75 \mathrm{~cm}^{-1}$.

been done in Fig. 2(a). Finally, it is easy to miss the first of the rotational Raman lines under this elastic "tail" as has occurred in the $\mathrm{CO}_{2}$ spectrum where the $j=0$ line is hidden. The student may readily discover this, however, by carefully checking the line separations with the displacement of the first line from the origin according to the relationship for $\Delta j= \pm 2$ Raman scattering. See below.

\section{DOUBLE SPECTROMETER SPECTRA OF $\mathrm{H}_{2}$, $\mathrm{N}_{2}, \mathrm{O}_{2}$, AND $\mathrm{CO}_{2}$}

In the case of $\mathrm{CO}_{2}$ it is not possible to identify clearly the first two Raman peaks, even with background subtraction. Therefore, in order to better elucidate the details of the Raman spectra we obtained additional data with a $1 \mathrm{~m}$ double spectrometer (Jobin-Yvon U1000) ${ }^{9}$ and the direct beam from an argon laser (Spectra Physics 2020-5) ${ }^{10}$ focused with a $10 \mathrm{~cm}$ lens. The same scattering cell was used and the detection system was substantially similar except that the system used a Hamamatsu R943-02 photomultiplier ${ }^{11}$ which has extended red response (work function $\sim 1.4 \mathrm{eV}$ ) and therefore must be cooled to $-30^{\circ} \mathrm{C}$ to avoid high thermionic emission "dark counts." The laser beam was focused with a 

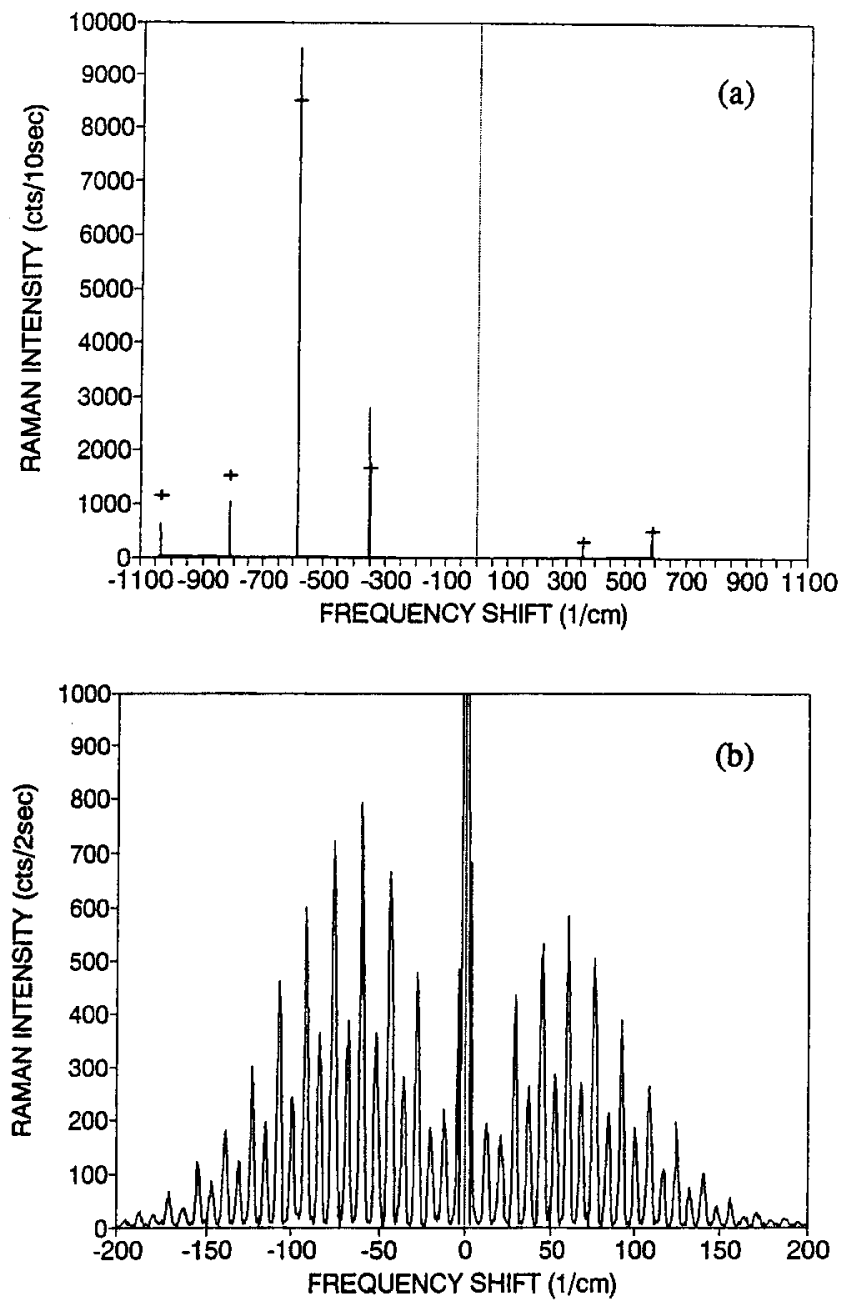

Fig. 3. Rotational Raman spectra obtained with a double spectrometer using $514.5 \mathrm{~nm}$ excitation and $300 \mu \mathrm{m}$ slits. (a) $\mathrm{H}_{2}$ gas at $1 \mathrm{~atm}, 200 \mathrm{~mW}$ power; (b) $\mathrm{N}_{2}$ gas at $1 \mathrm{~atm}, 120 \mathrm{~mW}$ power. In (a) the crosses mark the intensities calculated from Eq. (8) with $T_{\text {rot }}=300 \mathrm{~K}$ and one overall intensity parameter. Because the rotational shifts are large, the alternating intensities are nearly obscured by the Boltzmann factor.

$10 \mathrm{~cm}$ lens to a beam waist of about $15 \mu \mathrm{m}$. The results are shown in Fig. 3, panels (a) and (b), for two gases which give alternating rotational intensities, $\mathrm{H}_{2}$ and $\mathrm{N}_{2}$, and in Fig. 4 panels (a) and (b) for two gases in which alternate lines are missing, $\mathrm{O}_{2}$ and $\mathrm{CO}_{2}$.

\section{ANALYSIS OF THE PURE ROTATIONAL RAMAN SPECTRA}

\section{A. The Raman shifts}

The energy of a classical rigid rotator is given by

$$
E=(1 / 2) I \omega^{2}=J^{2} / 2 I \text { (joules), }
$$

where $J$ is the angular momentum and $I=\mu R^{2}$ is the rotational inertia. For all cases studied here, the reduced mass is $\mu=M / 2$ since for $\mathrm{CO}_{2}$ the carbon atom lies at the center of mass (center of rotation). Thus $R$ is the internuclear separation for the diatomic molecules and the oxygen separation for $\mathrm{CO}_{2}$.

With the quantization of angular momentum

$$
J^{2}=\left(h^{2} / 4 \pi^{2}\right) j(j+1) \text {, }
$$
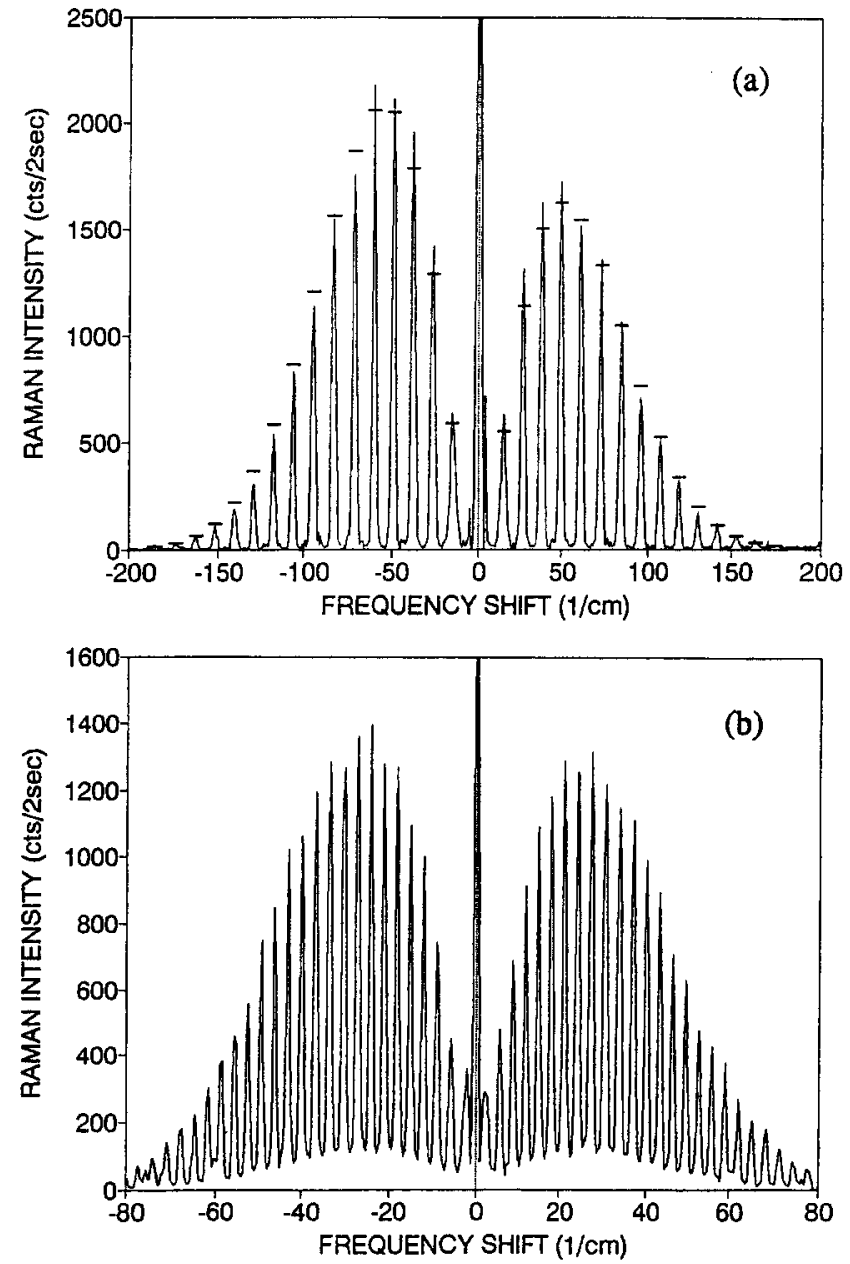

Fig. 4. Rotational Raman spectra obtained with a double spectrometer and $120 \mathrm{~mW}$ of laser power at $514.5 \mathrm{~nm}$. (a) $\mathrm{O}_{2}$ gas at $1 \mathrm{~atm}$, with $300 \mu \mathrm{m}$ slits; (b) $\mathrm{CO}_{2}$ gas at $1 \mathrm{~atm}$, with $100 \mu \mathrm{m}$ slits. In (a) horizontal bars indicate the intensities calculated from Eq. (8) using one adjustable intensity parameter and $T_{\mathrm{rot}}=300 \mathrm{~K}$.

we obtain the familiar energy levels of the quantized rotator

$$
E_{j}=\left[h^{2} /\left(8 \pi^{2} I\right)\right] j(j+1) \text { (joules). }
$$

For Raman scattering the angular momentum selection rule follows from two successive electric dipole transitions

$$
\Delta j=0, \pm 2 \text {. }
$$

Of course the $\Delta j=0$ transition is simply part of the Rayleigh peak. Thus the frequencies, in units of $\mathrm{cm}^{-1}$ or wave numbers, of the pure rotational Raman lines are given by

$$
\begin{aligned}
\Delta \bar{\nu} & =\Delta E_{j} / h c=\left(E_{j+2}-E_{j}\right) / h c \\
& =\left[h /\left(8 \pi^{2} c I\right)\right](4 j+6) \\
& =B(4 j+6)\left(\mathrm{cm}^{-1}\right),
\end{aligned}
$$

where $B$ is called the rotational constant ${ }^{2}$ and the speed of light $c$ must be in $\mathrm{cm} / \mathrm{s}$. Thus the spectrum consists of lines spaced equally by $4 B$ and the first line is displaced from the laser line by the frequency $6 B$, provided all rotational states are allowed. In fact, $\mathrm{O}_{2}$ and $\mathrm{CO}_{2}$ are examples in which half of the rotational states are forbidden by the symmetry of the overall molecular wave function under exchange of the nuclei. These effects are reviewed below. 


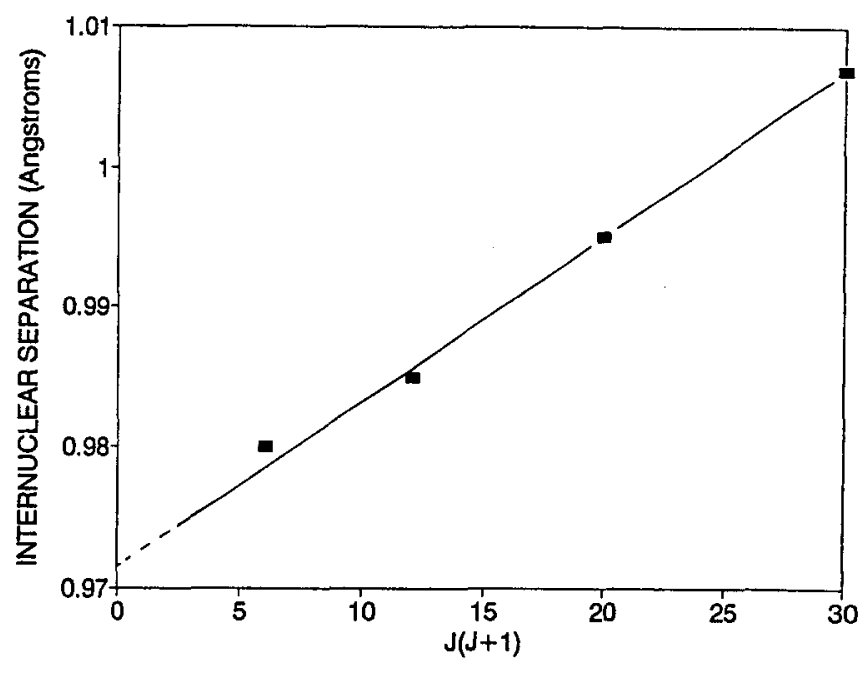

Fig. 5. Change of internuclear separation in $\mathrm{H}_{2}$ as a function of the quantity $j(j+1)$, where $j$ is the rotational quantum of the final state in the Stokes spectrum of Fig. 2(a).

\section{B. Exchange symmetry}

It is well known that for $\mathrm{H}_{2}$ there are ortho and para forms of the molecule depending on whether the total nuclear spin of the molecule $i=1$ or 0 , respectively. ${ }^{2,12,13}$ The nuclear spin of the molecule and the required symmetry under exchange of the nuclei also determine the allowed rotational states. Thus for ortho $\mathrm{H}_{2}$ for example, only odd values of the rotational quantum $j$ are permitted. The physics is discussed in a number of texts. ${ }^{12,13}$ For completeness we provide a summary of the argument below.

The total molecular wave function $\Psi_{\text {tot }}$ may be written in the customary way as a product wave function

$$
\begin{aligned}
\Psi_{\text {tot }}\left(R_{1} R_{2} r_{a} r_{b} I_{1} I_{2} s_{a} s_{b}\right)= & \psi_{e}\left(r_{a} r_{b}\right) \chi_{e}\left(s_{a} s_{b}\right) \\
& \times \psi_{n}\left(R_{1} R_{2}\right) \chi_{n}\left(I_{2} I_{2}\right),
\end{aligned}
$$

where $\psi_{e}\left(r_{a} r_{b}\right)$ is the electronic space part of the molecular wave function with electron coordinates $r_{a}$ and $r_{b}, \chi_{e}\left(s_{a} s_{b}\right)$ is the electronic spin part of the molecular wave function with electron spins $s_{a}$ and $s_{b}, \psi_{n}\left(R_{1} R_{2}\right)$ is the nuclear space part of the molecular wave function with $R_{1}$ and $R_{2}$ as the two nuclear coordinates, and $\chi_{n}\left(I_{1} I_{2}\right)$ is the nuclear spin part of the molecular wave function with $I_{1}$ and $I_{2}$ as the two nuclear spins.

The space component $\psi_{n}$ of the nuclear part of the wave function is a product of a vibrational term $\psi_{n}^{\text {vib }}$ and a rotational term $\psi_{n}^{\text {rot }}$; however, the vibrational term is always symmetric under nuclear exchange ${ }^{2,12}$ so it may be ignored, especially since we are always concerned with the ground vibrational state in pure rotational Raman scattering.

A most important property, for the Raman analysis, is the symmetry of the wave function under exchange of the two nuclei. The overall wave function must satisfy the appropriate exchange property of fermions in the case of the proton exchange for the molecule $\mathrm{H}_{2}$, or the exchange property of bosons for the cases of ${ }^{16} \mathrm{O}$ exchange $\left(\mathrm{O}_{2}\right.$ and $\left.\mathrm{CO}_{2}\right)$ and ${ }^{14} \mathrm{~N}$ exchange $\left(\mathrm{N}_{2}\right)$.

Thus for $\mathrm{H}_{2}$, the overall wave function must be antisymmetric under exchange of the two protons. Since both the space and spin parts of the electronic molecular wave function $\left({ }^{1} \Sigma_{g}^{+}\right)$are symmetric under this operation, two types of molecules exist. If the total molecular nuclear spin is 1 , then the nuclear spin wave function is even under this symmetry operation and the space part of the nuclear wave function must be odd under proton exchange. Since the vibrational part is always symmetric under this operation, the rotational part must be antisymmetric. The symmetry of the rotational part under the exchange operation is

$$
\psi_{\text {rot }}\left(R_{1}, R_{2}\right)=(-1)^{j} \psi_{\text {rot }}\left(R_{2}, R_{1}\right) .
$$

Thus one concludes that for $i_{\text {tot }}=1$, only states of odd $j$ are allowed. These states with aligned nuclear spin are known as ortho-hydrogen and have a spin multiplicity of $(2 i+1)=3$. Similar arguments for the case of para-hydrogen $\left(i_{\text {tot }}=0\right)$ show that the rotational part of the wave function must be symmetric since the nuclear spin part of the molecular wave function is antisymmetric. Thus the even rotational states of para-hydrogen have a spin multiplicity of $(2 i+1)=1$ and we expect a three-to-one ratio of intensities for odd versus even $j$ values in the Raman scattering spectrum. In the case of $\mathrm{H}_{2}$, the rotational inertia is small and correspondingly the rotational constant $B$ is large $\left(B=59 \mathrm{~cm}^{-1}\right)$ so that this $3: 1$ intensity ratio is partly obscured by the Boltzmann population factor. [See Fig. 3(a).]

The analyses for $\mathrm{O}_{2}, \mathrm{CO}_{2}$, and $\mathrm{N}_{2}$ proceed in a similar way. As seen for the case of $\mathrm{H}_{2}$, the known symmetry of the space and spin parts of the electronic wave function of the molecule allows one to focus on the symmetry of the product of the rotational and spin wave functions of the nuclei under the operation of nuclear exchange. It is generally true that the electronic ground state molecular wave function is symmetric under nuclear exchange; however, $\mathrm{O}_{2}$ is an important counterexample in which the electronic ground state $\left({ }^{3} \Sigma_{g}^{-}\right)$is odd under this operation. (The electronic ground state of $\mathrm{CO}_{2}$ has the more usual even symmetry, however.) Thus the following situations occur.

(1) For $\mathrm{O}_{2}$, the ${ }^{16} \mathrm{O}$ nuclei are "even-even" (in fact, doubly magic) and thus have spin $i=0$, making them bosons. The overall molecular wave function must therefore be even under the exchange of nuclei. Since the electronic part of the molecular wave function is known to be antisymmetric under this exchange, ${ }^{2}$ the nuclear part must also be antisymmetric. Since the total molecular nuclear spin wave function can only be symmetric ( $i=0$ and $i_{\text {tot }}=0$ ), therefore only odd $j$ values are allowed. The Raman spectrum will have all even $j$ values missing. See Fig. 4(a).

(2) For $\mathrm{CO}_{2}$, since it is a linear molecule with the carbon at the center of mass, the nuclear exchange operation is equivalent to that for $\mathrm{O}_{2}$. However, the electronic part of the molecular wave function is symmetric under this exchange. Thus only even $j$ values will be allowed and the Raman spectrum will have all odd $j$ values missing. See Fig. 4(b).

(3) For $\mathrm{N}_{2}$, the ${ }^{14} \mathrm{~N}$ nuclei have spin $i=1$ so that the molecular spin has three possible values, $i_{\text {tot }}=0,1,2$. With the electronic part of the molecular wave function being even under nuclear exchange, the nuclear part must be even as well. (Since the ${ }^{14} \mathrm{~N}$ nuclei have integer spin, they are Bosons.) When the total molecular nuclear spin is maximum $\left(i_{\text {tot }}=2\right)$, the spin part of the wave function is symmetric under exchange and therefore the rotational part must also be symmetric, i.e., only even $j$ values are permitted. With $i_{\text {tot }}=1$, the nuclear spin wave function is antisymmetric and for this case only odd $j$ values are allowed. Finally with $i_{\text {tot }}=0$, the nuclear spin wave function is symmetric again and even $j$ values are required. This leads to the following 
for ${ }^{14} \mathrm{~N}_{2}$ : (a) even $j$ values arise from both $i_{\text {tot }}=2$ and 0 with a total spin multiplicity of $\Sigma\left(2 i_{\text {tot }}+1\right)=5+1=6$; and (b) odd $j$ values arise from $i_{\text {tot }}=1$ with a spin multiplicity of $\left(2 i_{\text {tot }}+1\right)=3$.

As a consequence one observes intensities alternating by a factor of 2 between the even- $j$ and odd $-j$ terms of the Raman spectrum. See Fig. 3(b).

With careful observations, the student will be able to verify these symmetry selection rules. Assuming that the first Raman line (nearest the laser line) is clearly distinguished, one need only measure carefully the line separations. According to Eq. (7), the following must occur. (1) If all $j$ values are allowed, the first Raman line lies at $6 B$ and the separation of adjacent lines is $4 B .\left(\mathrm{H}_{2}\right.$ and $\left.\mathrm{N}_{2}\right)$. (2) If only odd $j$ values are allowed, the first line will be displaced from the laser line by $10 B$ and the separations of adjacent lines will be $8 B$. (3) If only even $j$ values are allowed, the first line will be displaced from the laser line by $6 B$ and the separations of adjacent lines will be $8 B$. From these relationships the student may independently verify the arguments outlined above-confirming the nuclear spin and/or the symmetry of the molecular electronic wave function.

\section{Rotational temperature}

The observation of the full rotational Raman spectrum provides an excellent opportunity for students to address the issue of the various factors which combine to yield the line intensities. In addition this provides an opportunity to discuss the concept of temperature and to introduce the idea of possible differences between the translational, rotational, and vibrational temperatures.

Understanding the overall shape of the rotational Raman spectra involves the nuclear spin and rotational spin multiplicities and, of course, the Boltzmann population distribution. Since the gas density is very low, one may treat this as an ideal, noninteracting gas in the classic sense. Since the electronic excited states allowed by dipole transitions are well into the ultraviolet range of the spectrum, there are no significant resonant Raman effects and the matrix elements are constant for all the rotational lines. Thus the photon scattering rate $R_{j}$ of any Raman line which involves the initial rotational state $j$ is simply proportional to the population of this state. Thus the photon scattering rate is ${ }^{14}$

$$
R_{j} \approx \nu_{S}^{3}\left(2 i_{\text {tot }}+1\right)(2 j+1) \exp \left(-E_{j} / k T\right), \text { (Stokes) }
$$

where $\nu_{S}$ is the Stokes frequency and $E_{j}$ is given by Eq. (1). The $\nu^{3}$ factor occurs with photon counting instead of the usual $\nu^{4}$ factor for intensities. Note that for the anti-Stokes lines the $j$ of the initial state is higher by two than the $j$ of the initial state for the Stokes lines. Thus the first anti-Stokes Raman line represents the transition from $j=2$ to $j=0$. Furthermore, in the anti-Stokes case, the angular momentum selection rules restrict the range of $j$ values so that multiplicity prefactor $(2 j+1)$ will indicate the multiplicity of the final state (the smaller of the initial and final state $j$ ). Thus the anti-Stokes photon scattering rate is

$$
R_{j} \approx \nu_{\mathrm{aS}}^{3}\left(2 i_{\mathrm{tot}}+1\right)(2 j+1) \exp \left(-E_{j+2} / k T\right) \text {, (anti-Stokes) }
$$

where $j$ is the value in the final (lower) state. To illustrate the temperature effects we have fit the functions of Eq. (8) to the data for the case of $\mathrm{O}_{2}$. The short horizontal lines give the fitted points for a rotational temperature of $300 \mathrm{~K}$.
Table I. $\Delta \tilde{\nu}_{j}$ and $R_{j}$ for $\mathrm{H}_{2}$.

\begin{tabular}{ccccc}
\hline \hline$j$ & 0 & 1 & 2 & 3 \\
\hline$\Delta \bar{\nu}_{j}\left(\mathrm{~cm}^{-1}\right)$ & 354.0 & 588 & 814.5 & 1035 \\
$R_{j}(\AA)$ & 0.980 & 0.985 & 0.995 & 1.007 \\
\hline \hline
\end{tabular}

We emphasize that the energy of the initial rotational state, $\left(E_{j}\right.$ or $\left.E_{j+2}\right)$ ought not to be confused with the energy shift $h c \Delta \bar{\nu}_{j}$ observed in the Raman spectrum. We have found this to be a common student mistake.

\section{STRETCHING OF $\mathrm{H}_{2}$ DUE TO CENTRIPETAL FORCES}

In the case of $\mathrm{H}_{2}$, with small rotational inertia, the rotational energies or velocities are large enough, even for small $j$, that some stretching of the molecule is readily observed in the Raman spectra. Although the exact line positions cannot be read with sufficient precision from Fig. 4(a), the numerical data obtained with $0.25 \mathrm{~cm}^{-1}$ steps yield the values for the positions as shown in Table I. The last row of Table I gives the value for the internuclear separation from $\mathrm{Eq}$. (5). It may readily be shown that the stretching $\Delta R_{j}$ is given, to a good approximation, by

$$
\Delta R_{j}=2 h c B j(j+1) / R k,
$$

where $k$ is the "spring constant" for the molecular binding. The data, as seen in Fig. 5, fit Eq. (10) quite well with the constant $k \approx 300 \mathrm{~N} / \mathrm{m}$.

The data for the other gases studied here also indicate some stretching with increasing rotational quantum number but the precision is not as good.

\section{CONCLUSIONS}

Partly because the rotational Raman spectra of simple molecules are straightforward and "well understood," they can provide a rich learning experience for students in the laboratory. We have shown that these spectra can be obtained with ordinary single spectrometers and modest power laser sources such as air-cooled argon lasers or with laser beams "borrowed" from a research lab via an optical fiber. Analysis of the spectra of $\mathrm{H}_{2}, \mathrm{O}_{2}, \mathrm{CO}_{2}$, and $\mathrm{N}_{2}$ yield the internuclear separations, the striking effects of the symmetry properties of the molecular wave function under exchange of the nuclei, and the measurement of rotational temperatures in the gas. Additionally, the student may measure the internuclear stretching due to centripetal force in the rotating $\mathrm{H}_{2}$ molecule.

Pure rotational Raman spectra are currently used in atmospheric and combustion research for measurements of temperature and density using techniques such as laser doppler radar and coherent anti-Stokes Raman scattering (CARS and inverse Raman). Thus the study of pure rotational Raman spectra, while illustrating some fundamentally important physics concepts, also provides an introduction to a class of valuable contemporary research methods.

Finally, it should be noted that Raman scattering in transparent liquids also may readily be done with a single spectrometer and low laser powers. (In fact the spectrometer may even be replaced by a simple diffraction grating in this case. $)^{15}$ Furthermore the use of colloidal or holographic notch filters, to attenuate the laser line, may also permit the 
use of single spectrometers for work on opaque solids. However, the rejection bandwidth of such notch filters is typically $200 \AA^{4}$ so that they are not suitable for small frequency shift studies such as pure rotational Raman scattering.

\section{ACKNOWLEDGMENTS}

A.D.C. wishes to acknowledge the hospitality of Bilkent University and the support of the NATO-D (Distinguished Senior Visitor) Program of the Scientific and Technical Research Council of Turkey (TUBITAK) during his collaboration at Bilkent U. where this work was completed. At Bilkent the assistance of Talal Azfar and Gökhan Ulu was greatly appreciated.

\footnotetext{
${ }^{1}$ See for example, Light Scattering in Solids, edited by M. Cardona, Topics in Applied Physics, Vols. 1-4 (Springer, Berlin, 1983, 1982, 1983, 1984, respectively); S. K. Freeman, Applications of Laser Raman Spectroscopy (Wiley, New York, 1974).

${ }^{2}$ G. Herzberg, Molecular Spectra and Molecular Structure, Spectra of Diatomic Molecules, Vol. 1, 2nd ed. (Van Nostrand, Princeton, 1950), Chap. III; G. Herzberg, Molecular Spectra and Molecular Structure, Infrared and
}

Raman Spectra of Polyatomic Molecules, Vol. 2 (Van Nostrand, New York, 1945), Chap. I.

${ }^{3}$ Raman Spectroscopy of Gases and Liquids, edited by A. Weber, Topics in Current Physics, Vol. 11 (Springer, Berlin, 1979), Chap. 3, pp. 71-121.

${ }^{4}$ Spex Industries, 3880 Park Ave, Edison, NJ 08820.

${ }^{5}$ Lexel Laser Inc, 48503 Milmont Dr., Fremont, CA 94538.

${ }^{6}$ Thorn-EMI Electron Tubes Inc., 23 Madison Rd., Fairfield, NJ 07006.

${ }^{7}$ Mechtronics Corp., 1701 N. 25th Ave., Melrose Park, IL 60160.

${ }^{8}$ B\&C Microsystems Inc., 355 West Olive Ave., Sunnyvale, CA.

${ }^{9}$ Jobin Yvon, 16/18 rue du Canal, BP118, 91163 Longjumeau Cedex, France.

${ }^{10}$ Spectra Physics, 1250 W. Middlefield Rd., P.O. Box 7013, Mountain View, CA 94039.

${ }^{11}$ Hamamatsu Photonics, 360 Foothill Rd., Bridgewater, NJ 08807.

${ }^{12}$ R. Eisberg and R. Resnick, Quantum Physics of Atoms, Molecules, Solids, Nuclei, and Particles, 2nd ed. (Wiley, New York, 1985), pp. 423-437.

${ }^{13}$ R. B. Leighton, Principles of Modern Physics (McGraw-Hill, New York, 1959), Chap. 9.

${ }^{14} \mathrm{~A}$. Compaan, "Resonance Raman scattering with tunable lasers," Appl. Spectrosc. Rev. 13, 295-369 (1977); A. Compaan and H. J. Trodahl, "Resonance Raman scattering in Si at elevated temperatures," Phys. Rev. B 29, 793-801 (1984).

${ }^{15}$ R. Feinberg, "A simple apparatus for observing the Raman effect," Am. J. Phys. 58, 893 (1990).

\title{
Multiloop dc circuits by source conversion and nodal analysis
}

\author{
David A. Giltinan \\ Department of Physics and Technology, Edinboro University of Pennsylvania, Edinboro, \\ Pennsylvania 16444
}

(Received 15 November 1993; accepted 17 January 1994)

A supplemental method for teaching multiloop dc circuits is presented. The use of source conversion and nodal analysis results in algebraically simpler equations than the conventional approach of most introductory calculus based physics texts.

In examining many of the standard introductory calculus based physics texts, one notes that dc circuits are solved in the same manner. The conventional method suggested for multiloop circuits is to assume branch currents, apply Kirchhoff's voltage law (KVL) to a number of the loops and Kirchhoff's current law (KCL) to a number of junction points, and thus obtain the sufficient number of equations to solve for the unknown branch currents. With this method a two loop circuit requires three simultaneous equations for the three branch currents. Using mesh currents will reduce the number of unknowns to two. When assigning problems involving three or more loops, it is usual to restrict one or more of the circuit parameters so that symmetry arguments can be used to reduce the complexity.

The purpose of this note is to suggest that physics teachers might consider the alternate representation of sources as ideal current sources, in parallel with internal resistances, and not restrict discussion to the usual representation as an ideal battery in series with an internal resistance. By Norton's or Thévenin's theorems, any linear source can be represented in either fashion. This alternate "source conversion" method ${ }^{1}$ allows one to replace a battery (emf $E$ and series internal resistance $r$ ), with a constant current source of value $I^{\prime}=E / r$ in parallel with a resistance of $r$. When followed by nodal analysis, it not only results in simpler algebra (no simultaneous equations for the two loop circuit) but it suggests to the students, some of whom may be engineering majors, that more efficient methods of solutions are available. While it should not necessarily be our intent to introduce circuit theorems such as Thévenin's, substitution, reciprocity and the like, a lot could be said for nodal analysis. It forms the basis of many circuit-solving software programs.

Applied to the simple two loop circuit of Fig. 1, the con-

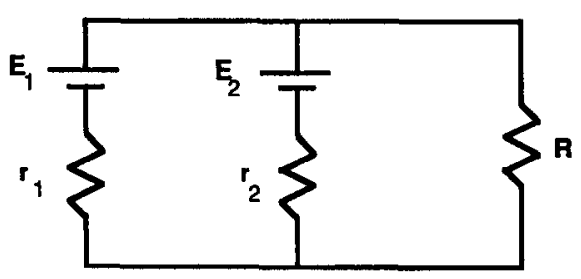

Fig. 1. Simple two-loop dc circuit, with two batteries having emfs $E_{1}$ and $E_{2}$ and internal resistances $r_{1}$ and $r_{2}$. 